\title{
Avaliação do estado nutricional da comunidade indígena Parkatêjê, Bom Jesus do Tocantins, Pará, Brasil
}

\author{
Evaluation of the nutritional status \\ of the Parkatêjê indigenous community \\ in Bom Jesus do Tocantins, Pará, Brazil
}

Jane de Carlos Santana Capelli 1,2

Sergio Koifman 2

\footnotetext{
1 Departamento de

Enfermagem,

Universidade Iguaçu.

Av. Abílio Augusto Távora

2134, Nova Iguaçu, RJ

26260-000, Brasil.

2 Departamento de

Epidemiologia e Métodos

Quantitativos em Saúde.

Escola Nacional

de Saúde Pública,

Fundação Oswaldo Cruz.

Rua Leopoldo Bulhões 1480 ,

80 andar, Rio de Janeiro, $R J$

21041-210, Brasil.
}

\begin{abstract}
The nutritional status of the Mãe-Maria indigenous community in Bom Jesus do Tocantins, Pará State, Brazil, was ascertained in a descriptive study in which ninety percent of the total population (278 individuals) agreed to participate. Weight-for-height and height-for-age indices and Body Mass Index (BMI) were ascertained for children and adults by gender, respectively, as were weight and height means in adolescents. Compared to NCHS curves, overweight and chronic malnutrition were observed, respectively, in 6.7 and $8.6 \%$ of all children under 10 years old (104). Weight means were similar among Indians and NCHS adolescents, while height means were lower among the former. Overweight prevalence (BMI 25 to 29) was $23.7 \%$ among male adults and 50.0\% among female adults, and obesity (BMI > 30) was observed in 12.5\% of adult females.
\end{abstract}

Key words Anthropometry; Nutrition; Obesity; Parkatêjê; South American Indians

Resumo Com o objetivo de conhecer o estado nutricional dos membros da Área Indígena MãeMaria em Bom Jesus do Tocantins, Pará, um estudo descritivo foi realizado com a participação de 90,8\% (278 pessoas) de seus integrantes. Os indicadores de massa corporal conforme estatura e de estatura segundo idade, bem como o índice de massa corporal consoante sexo (IMC), foram determinados para crianças e adultos, respectivamente, além das médias de peso e estatura em adolescentes. Comparativamente às curvas normatizadas da população norte-americana (NCHS), notou-se prevalência de sobrepeso e de desnutrição crônica da ordem de 6,7 e 8,6\%, respectivamente, nas 104 crianças menores de dez anos avaliadas. As médias de peso encontradas nos adolescentes indígenas e na população NCHS foram relativamente similares, sendo as médias de estatura menores entre os primeiros. A prevalência de sobrepeso (IMC 25-29) em homens e mulheres adultos foi de 23,7 e 50,0\%, respectivamente, detectando-se prevalência de obesidade (IMC > 30) da ordem de 12,5\% entre as últimas.

Palavras-chave Antropometria; Nutrição; Obesidade; Parkatêjế, Índios Sul-americanos 


\section{Introdução}

O interesse em avaliar o estado nutricional de sociedades indígenas distribuídas pelo território nacional não é recente. Contudo, é relativamente pequeno o número de trabalhos realizados neste segmento específico da população brasileira que visem conhecer o seu perfil de saúde e de nutrição e, em especial, seus padrões de crescimento físico, ou que busquem caracterizar o impacto acarretado pelas mudanças sócio-econômicas, ambientais e culturais introduzidas desde o contato com a sociedade nacional brasileira (Coimbra Jr. \& Santos, 1991). A partir da década de noventa, entretanto, vemse notando o fortalecimento de pesquisas na área da epidemiologia nutricional voltadas às comunidades indígenas no Brasil, em que há associação de dados obtidos através de inquéritos antropométricos e descrição dos meios de subsistência com os padrões de morbi-mortalidade (Gugelmin, 1995; Leite, 1998; Santos \& Coimbra Jr., 1996; Tavares et al., 1999 a, 1999b).

Em 1994 foi feito um estudo exploratório na comunidade indígena Parkatêjê - que vive no Município de Bom Jesus do Tocantins, Pará (Koifman et al., 1998) -, com o objetivo de avaliar o impacto de diferentes exposições ambientais, associadas possivelmente ao inusitado aparecimento de casos de câncer em adultos jovens desta população. Durante o curso de sua realização, foi desenvolvida uma avaliação antropométrica, pois nenhum estudo similar havia sido efetuado anteriormente nessa comunidade. Assim, o presente trabalho tem como objetivo apresentar os resultados obtidos na avaliação antropométrica da comunidade indígena Parkatêjê, realizada naquela oportunidade, comparando tais resultados com aqueles obtidos em estudos similares feitos em outras comunidades indígenas.

\section{Materiais e métodos}

A coleta de dados foi realizada na comunidade indígena Parkatêjê, que era constituída, em outubro de 1994, por 306 habitantes. Aqueles que concordaram em participar da investigação (278 pessoas; $90,8 \%$ ) foram pesados e medidos por um dos autores (JCSC), previamente treinado. As medidas de massa corporal, comprimento e estatura foram obtidas de todas as 104 crianças menores de nove anos, inclusive; de 75 adolescentes entre 10 e 19 anos; de 99 adultos maiores de 20 anos, como também foram coletadas as variáveis sexo e idade. As idades das crianças, adolescentes e adultos jovens foram copia- das de um livro de registro de nascimentos disponível no posto da FUNAI (Fundação Nacional do Índio) situado na própria aldeia indígena. Os 36 adultos acima de 40 anos avaliados tinham idades estimadas em virtude da ausência de registro das respectivas datas de nascimento.

Utilizou-se uma balança microeletrônica, marca Filizola - com capacidade de $150 \mathrm{~kg}$ e resolução de $100 \mathrm{~g}$ - para obtenção do valor da medida de massa corporal; um antropômetro de campo foi usado para medição do comprimento de crianças menores de dois anos (posição em decúbito dorsal) e, para a medição da estatura de crianças maiores de dois anos (posição vertical), foi empregada uma fita métrica marca Stanley francesa, com resolução de $3 \mathrm{~mm}$.

Os dados antropométricos das crianças foram agrupados nos índices de massa corporal para estatura (MC/EST) e estatura para idade (EST/I), recorrendo-se à proporção de desvio padrão em relação à mediana (escore z) de referência das curvas normatizadas da população norte-americana (NCHS, 1977). Seguindo a Organização Mundial da Saúde (WHO, 1995), estabeleceu-se o ponto de corte de -2 desvios padrão dos índices de MC/EST e EST/I para diferenciar crianças eutróficas das desnutridas. Assim sendo, as crianças que tiveram desvios padrão de MC/EST abaixo de - 2 foram classificadas como apresentando baixo peso - ou desnutrição aguda ou atrofia nutricional -, e aqueles abaixo de - 2 para o indicador de EST/I, como evidenciando desnutrição crônica - ou nanismo nutricional. As que apresentaram desvios padrão de MC/EST acima de +2 foram classificadas como tendo sobrepeso, e as que exibiram medidas de MC/EST e EST/I compreendidas entre $-1,99 \mathrm{a}+1,99$, como eutróficas. Foram calculadas as médias de massa corporal e estatura da população adolescente, analisadas segundo faixa etária e sexo, e comparadas com as médias das curvas de referência da população adolescente norte-americana do NCHS. Para a população adulta utilizou-se o Índice de Massa Corporal (IMC) através da razão entre massa corporal $(\mathrm{kg})$ versus estatura $(\mathrm{cm})$ elevada ao quadrado $\left(\mathrm{kg} / \mathrm{cm}^{2}\right)$ com pontos de corte definidos por Bray (1989): baixo peso (IMC < 20); adequação ( $20 \leq \mathrm{IMC}<25)$; sobrepeso $(25 \leq$ IMC $<30)$; obesidade (IMC $\geq 30$ ).

\section{Resultados}

Do total da população infantil estudada, observou-se que apenas uma criança se encontrava com baixo peso e sete crianças $(6,7 \%)$ apresentaram sobrepeso: uma criança na faixa etária 
de menores de um ano e seis crianças $(16,2 \%)$ na faixa etária compreendendo 6 a 9 anos (Tabela 1). Através da E/I, constatou-se que quatro crianças menores de dois anos $(22,2 \%$ do total das crianças analisadas na faixa etária) apresentaram desnutrição crônica (Tabela 2). Dentre as 58 crianças avaliadas, os achados demonstraram prevalência desta condição em 8,6\% das crianças menores de dez anos, não sendo observados déficits estaturais em crianças entre 2 a 5 anos.

As médias de massa corporal e estatura dos adolescentes indígenas Parkatêjê na faixa etária de 10-14 anos (Tabela 3) mostraram-se relativamente similares quando comparadas com as médias das curvas de referência da população adolescente normatizadas pelo NCHS. Os adolescentes de ambos os sexos na faixa etária de 15-19 apresentaram índices pondero-estaturais mais reduzidos que aqueles observados na população de referência do NCHS (respectivamente, $4 \mathrm{~kg}$ e $6,0 \mathrm{~cm}$ no sexo masculino, e $3 \mathrm{~kg}$ e 7,0 cm no sexo feminino).

Dos 99 adultos avaliados, 59,6\% eram do sexo masculino, notando-se concentração de adultos jovens (20 a 39 anos) em ambos os sexos. A prevalência de sobrepeso foi de $50,0 \%$ em mulheres e $23,7 \%$ em homens, e a de obesidade de $12,5 \%$ e $1,7 \%$, respectivamente (Tabela 4). Estes percentuais indicam que mais da metade da população feminina $(62,5 \%)$ mostrou excesso de massa corporal total. A média e a mediana mais elevadas do IMC segundo faixa etária em mulheres foram observadas na faixa etária de 50-59 anos (Tabela 5).

\section{Discussão}

Os Parkatêjê, grupo Jê - Timbíra, mais conhecidos como “Gavião de Mãe Maria”, vivem na Área Indígena Mãe Maria (AIMM) desde os meados de 1970. Os primeiros contatos e relações estabelecidas com as frentes de expansão da sociedade nacional brasileira aconteceram no final do século XIX (Ferraz, 1985), havendo, no início da década de 50, uma ruptura sócioeconômica importante, devido ao esfacelamento dos territórios comuns, doenças e diminuição de seu contingente populacional. Como estratégia de subsistência, os Parkatêjê desenvolveram a caça de animais, a venda de carnes e couros de animais de caça, a agricultura de subsistência e a coleta de frutos silvestres. Todavia, a introdução de alimentos industrializados em sua dieta vem aumentando progressivamente, como o óleo de soja, sal, açúcar, café, pão e biscoito, obtidos quando saem da aldeia (Iara Ferraz, comunicação pessoal).
Tabela 1

Distribuição de freqüências de massa corporal segundo faixa etária, crianças indígenas Parkatêjê (0-9 anos), 1994.

\begin{tabular}{|c|c|c|c|c|c|c|c|c|}
\hline \multirow{3}{*}{$\begin{array}{l}\text { Faixa Etária } \\
\text { (anos) }\end{array}$} & \multicolumn{8}{|c|}{ Massa Corporal segundo Estatura } \\
\hline & \multicolumn{2}{|c|}{ Baixo Peso } & \multicolumn{2}{|c|}{ Adequação } & \multicolumn{2}{|c|}{ Sobrepeso } & \multicolumn{2}{|c|}{ Total } \\
\hline & $\mathrm{n}$ & $\%$ & $\mathrm{n}$ & $\%$ & $\mathrm{n}$ & $\%$ & $\mathrm{n}$ & $\%$ \\
\hline $00-01$ & 00 & 0.0 & 14 & 93.3 & 01 & 6.7 & 15 & 100.0 \\
\hline $01-05$ & 01 & 2.0 & 51 & 98.0 & 00 & 0.0 & 52 & 100.0 \\
\hline $06-09$ & 00 & 0.0 & 31 & 83.8 & 06 & 16.2 & 37 & 100.0 \\
\hline Total & 01 & 1.0 & 96 & 92.3 & 07 & 6.7 & 104 & 100.0 \\
\hline
\end{tabular}

Tabela 2

Distribuição de freqüências de estatura por idade, crianças indígenas Parkatêjê (0-9 anos), 1994.

\begin{tabular}{|c|c|c|c|c|c|c|}
\hline \multirow{2}{*}{$\begin{array}{l}\text { Faixa Etária } \\
\text { (anos) }\end{array}$} & \multicolumn{2}{|c|}{$<-2 S$} & \multicolumn{2}{|c|}{$>-2 S-<2 S$} & \multicolumn{2}{|c|}{ Total } \\
\hline & $\mathrm{n}$ & $\%$ & $\mathrm{n}$ & $\%$ & $\mathrm{n}$ & $\%$ \\
\hline $00-01$ & 04 & 22.2 & 14 & 77.7 & 18 & 100.0 \\
\hline $02-05$ & 00 & 0.0 & 22 & 100.0 & 22 & 100.0 \\
\hline $06-09$ & 01 & 0.3 & 17 & 99.7 & 18 & 100.0 \\
\hline Total & 05 & 8.6 & 53 & 91.4 & 58 & 100.0 \\
\hline
\end{tabular}

Tabela 3

Distribuição de freqüências de massa corporal e estatura segundo sexo e faixa etária (10-15 anos) de adolescentes indígenas Parkatêjê (1994) e da população NCHS (1977).

\begin{tabular}{|c|c|c|c|c|c|c|c|}
\hline \multirow{3}{*}{$\begin{array}{l}\text { Faixa Etária } \\
\text { (anos) }\end{array}$} & \multirow[b]{3}{*}{$\mathrm{n}$} & \multicolumn{3}{|c|}{$M C(K g)$} & \multicolumn{3}{|c|}{ Estatura $(\mathrm{cm})$} \\
\hline & & \multicolumn{2}{|c|}{ Parkatêjê } & \multirow{2}{*}{$\begin{array}{l}\text { NCHS } \\
\text { média }\end{array}$} & \multicolumn{2}{|c|}{ Parkatêjê } & \multirow{2}{*}{$\begin{array}{l}\mathrm{NCHS} \\
\text { média }\end{array}$} \\
\hline & & média & EP & & média & EP & \\
\hline \multicolumn{8}{|l|}{ Homens } \\
\hline $10-14$ & 20 & 43.9 & 2.9 & 43.7 & 152.2 & 2.7 & 151.8 \\
\hline $15-19$ & 21 & 63.1 & 1.7 & 67.1 & 168.6 & 1.0 & 174.5 \\
\hline \multicolumn{8}{|l|}{ Mulheres } \\
\hline $10-14$ & 24 & 46.1 & 2.1 & 44.4 & 149.9 & 1.5 & 151.2 \\
\hline $15-19$ & 09 & 54.9 & 2.1 & 57.9 & 155.6 & 1.5 & 162.9 \\
\hline
\end{tabular}

$\mathrm{EP}=$ erro padrão 
Tabela 4

Distribuição de freqüências do Índice de Massa Corporal segundo sexo, adultos indígenas Parkatêjê, outubro, 1994.

\begin{tabular}{|c|c|c|c|c|}
\hline \multirow[t]{2}{*}{ IMC (Kg/m2) } & \multicolumn{2}{|c|}{ M } & \multicolumn{2}{|c|}{$F$} \\
\hline & $n$ & $\%$ & $\mathrm{n}$ & $\%$ \\
\hline Baixo peso $(<20)$ & 1 & 1.7 & 2 & 5.0 \\
\hline Adequação (20 - 24) & 43 & 72.9 & 13 & 32.5 \\
\hline Sobrepeso (25 - 29) & 14 & 23.7 & 20 & 50.0 \\
\hline Obeso $(\geq 30)$ & 1 & 1.7 & 5 & 12.5 \\
\hline Total & 59 & 100.0 & 40 & 100.0 \\
\hline
\end{tabular}

Tabela 5

Distribuição de freqüências do Índice de Massa Corporal, segundo sexo e faixa etária, adultos indígenas Parkatêjê, 1994.

\begin{tabular}{|c|c|c|c|c|c|c|c|c|}
\hline \multirow[t]{2}{*}{ Faixa Etária } & \multicolumn{8}{|c|}{ Índice de Massa Corporal ( $\mathrm{Kg} /\left(\mathrm{m}^{2}\right)$} \\
\hline & $\mathrm{n}$ & $x$ & Z & Mediana & P25 & P75 & Min. & Máx. \\
\hline \multicolumn{9}{|l|}{ Homens } \\
\hline $20-29$ & 25 & 23.9 & 1.8 & 23.7 & 22.6 & 24.4 & 21.7 & 30.3 \\
\hline $30-39$ & 12 & 24.3 & 2.7 & 24.3 & 22.5 & 25.8 & 20.3 & 29.8 \\
\hline $40-49$ & 9 & 24.3 & 2.4 & 25.2 & 22.2 & 25.5 & 20.2 & 27.2 \\
\hline $50-59$ & 7 & 23.7 & 6.2 & 23.9 & 21.8 & 24.9 & 19.8 & 27.7 \\
\hline $60+$ & 6 & 25.7 & 1.9 & 25.6 & 24.3 & 27.2 & 23.4 & 28.2 \\
\hline \multicolumn{9}{|l|}{ Mulheres } \\
\hline $20-29$ & 13 & 25.3 & 4.2 & 25.3 & 23.0 & 28.0 & 17.3 & 33.8 \\
\hline $30-39$ & 13 & 26.9 & 4.4 & 26.9 & 24.4 & 29.9 & 18.4 & 34.8 \\
\hline $40-49$ & 7 & 25.8 & 3.2 & 25.9 & 23.2 & 28.4 & 21.3 & 31.0 \\
\hline $50-59$ & 2 & 28.9 & 0.4 & 28.9 & 28.6 & 29.1 & 28.6 & 29.1 \\
\hline $60+$ & 5 & 25.2 & 3.7 & 23.5 & 23.1 & 29.0 & 21.0 & 29.2 \\
\hline
\end{tabular}

$\mathrm{X}=$ Média; $Z$ = Desvio Padrão; $\mathrm{P} 25=$ Percentil 25; P75= Percentil 75

As crianças Parkatêjê menores de cinco anos apresentaram valores reduzidos de desnutrição crônica (10\%) quando comparados com a média nacional $(15,4 \%)$ (INAN, 1990). Inquéritos antropométricos realizados em crianças de outras comunidades indígenas, no Brasil, revelaram freqüências de baixa estatura para idade bastante expressivas, não raro superiores a 30\% (Coimbra Jr. \& Santos, 1991; Leite 1998; Weiss, 1998). Assim, observa-se que as crianças Parkatêjê não se enquadram no perfil predominante das crianças indígenas brasileiras, as quais se apresentam usualmente com a estatura abaixo da população de referência. Por outro lado, merece também ser ressaltada a prevalência de sobrepeso em crianças meno- res de dez anos $(6,7 \%)$, sobretudo nas crianças entre 6 e 9 anos (16,7\%).

Todos os adolescentes entre 15 e 19 anos apresentaram médias de massa corporal similares as do padrão de referência, sendo as médias de estatura inferiores (em torno de 7,0 cm) àquelas do padrão internacional (NCHS, 1977), não seguindo o comportamento achado em crianças menores de nove anos, que apresentaram baixo percentual para desnutrição crônica.

Na população adulta Parkatêjê foram observados elevados percentuais de sobrepeso e obesidade, principalmente a feminina. Ao compará-la a outras comunidades, observou-se percentuais similares para sobrepeso, como, por exemplo, o observado por Leite (1998), que encontrou $49,2 \%$ de sobrepeso ao avaliar o IMC de 63 mulheres adultas Xavánte da Aldeia de São José, e elevados percentuais em homens adultos Xavánte $(\mathrm{n}=66 ; 41,6 \%)$. Quanto à obesidade, a mesma população apresentou valores maiores que a Parkatêjê, $41,3 \%$ na feminina e $24,6 \%$ na masculina. Entretanto, Tavares et al. (1999b) encontraram $67,7 \%$ de sobrepeso ou obesidade na população adulta Parkatêjê, sendo que as maiores freqüências de alterações glicêmicas ocorreram entre as mulheres que tinham maior prevalência de sobrepeso. Ainda neste grupo foram observados 40 índios $(44,4 \%)$ cujos níveis lipídicos estavam fora dos valores desejáveis de acordo com o Consenso Brasileiro de Dislipidemia (Tavares et al., 1999a). Estes achados indicam tendência progressiva de ganho de massa corporal no período de 1994 a 1999, bem como o aparecimento de doenças associadas a esse perfil nutricional. Recentes estudos têm indicado o surgimento de patologias como diabetes mellitus tipo II, doenças cardiovasculares, entre outras, em alguns grupos indígenas, por estarem sofrendo um acelerado e importante processo de modificação em seus padrões sócio-econômicos e culturais, tais como meios de subsistência, dieta e padrões de atividade física (Santos \& Coimbra Jr., 1996; Vieira Filho, 1996). Assim, o que se observa na população Parkatêjê sugere um comportamento similar ao das demais populações estudadas por aqueles autores.

Conclui-se que estudos de avaliação nutricional englobando antropometria, consumo alimentar, exames laboratoriais, tendências de morbi-mortalidade devem ser realizados nesta comunidade, visando compreender este padrão diferenciado das demais comunidades indígenas brasileiras, já que este grupo vem sofrendo um processo rápido e intenso de modificação nos seus padrões sócio-econômicos, ambientais e culturais. 


\section{Agradecimentos}

À Profa. Dra. Iara Ferraz (Universidade Federal do Pará), ao Prof. Dr. Luiz Antonio dos Anjos, (Escola Nacional de Saúde Pública, Fundação Oswaldo Cruz), à Profa. Silvia Angela Gugelmin (Universidade do Estado do Rio de Janeiro) e Prof. Ricardo Santos (Escola Nacional de Saúde Pública, Fundação Oswaldo Cruz), pelas sugestões e revisão crítica dadas ao trabalho.

\section{Referências}

BRAY, G. A., 1989. Classification and evaluation of the obesities. Medical Clinics of North America, 73: 161-184.

COIMBRA Jr., C. E. A. \& SANTOS, R. V., 1991. Avaliação do estado nutricional num contexto de mudança sócio-econômica: O grupo indígena Suruí do Estado de Rondônia, Brasil. Cadernos de Saúde Pública, 7:538-562.

FERRAZ, I., 1985. Gavião. In: Povos Indígenas no Brasil (Centro Ecumênico de Documentação e Informação, org.), pp. 53-99, São Paulo: Centro Ecumênico de Documentação e Informação.

GUGELMIN, S. A., 1995. Nutrição e Alocação de Tempo dos Xavánte de Pimentel Barbosa, Mato Grosso: Um Estudo sobre Mudanças. Dissertação de Mestrado, Rio de Janeiro: Escola Nacional de Saúde Pública, Fundação Oswaldo Cruz.

INAN (Instituto Nacional de Alimentação e Nutrição), 1990. Pesquisa Nacional sobre Saúde e Nutrição, PNSN - 1989. Resultados Preliminares. Brasília, DF: Instituto Nacional de Alimentação e Nutrição, Ministério da Saúde.

KOIFMAN, S.; FERRAZ, I.; VIANA, T. S.; SILVEIRA, C. L. P.; CARNEIRO, M. T. D.; KOIFMAN, R. J.; SARCINELLI, P. N.; MATTOS, R. C. O. C.; LIMA, J. S.; SILVA, J. J. O.; MOREIRA, J. C.; FERREIRA, M. F. A.; FERNANDES, C. \& BULCÃO, A. C., 1998. Agrupação de casos de câncer em adultos jovens indígenas residindo nas proximidades de linhas de transmissão de alta tensão elétrica em Bom Jesus do Tocantins, Pará, Brasil. Cadernos de Saúde Pública, 14 (Sup. 3):161-172.

LEITE, M. S., 1998. Avaliação do Estado Nutricional da População Xavánte de Sangradouro-Volta Grande, Mato Grosso. Dissertação de Mestrado, Rio de Janeiro: Escola Nacional de Saúde Pública, Fundação Oswaldo Cruz.
NCHS (National Center for Health Statistics), 1977. NCHS Growth Curves for Children Birth - 18 Years United States. Vital and Health Statistics Series 11, No. 165. Washington, DC: U.S. Printing Office.

SANTOS, R. V. \& COIMBRA Jr., C. E. A., 1996. Socioeconomic differentiation and body morphology in the Surui of Southwestern Amazonia. Current Antrhopology, 37:851-856.

TAVARES, E. F.; VIEIRA FILHO, J. P. B.; ANDRIOLO, A. \& FRANCO, L. J., 1999a. Anormalidades de tolerância à glicose e fatores de risco cardiovascular em uma tribo indígena aculturada da região brasileira. Arquivos Brasileiros de Endocrinologia e Metabologia, 43 (Sup. 1):S235.

TAVARES, E. F; VIEIRA FILHO, J. P. B.; ANDRIOLO, A. \& FRANCO, L. J., 1999b. Níveis de insulina, próinsulina e anti-GAD 65 na população indígena Parkatêjê. Arquivos Brasileiros de Endocrinologia e Metabologia, 43 (Sup. 1):S248.

VIEIRA FILHO, J. P. B., 1996. Emergência do diabetes melito tipo II entre os Xavánte. Revista da Associação Médica Brasileira. 42:61.

WEISS, M. C. V., 1998. Contato Interétnico, Perfil de Saúde e Doença e Modelos de Intervenção: O Caso dos Enawenê-nawê em Mato-Grosso. Tese de Doutorado, Rio de Janeiro: Escola Nacional de Saúde Pública, Fundação Oswaldo Cruz.

WHO (World Health Organization), 1995. Physical Status: The Use and Interpretation of Anthropometry. Technical Report Series 854. Geneva: WHO. 